\title{
Inflammation, Oxidative Stress, and Antioxidants Contribute to Selected Sleep Quality and Cardiometabolic Health Relationships: A Cross-Sectional Study
}

\author{
Thirumagal Kanagasabai and Chris I. Ardern \\ School of Kinesiology and Health Science, York University, Toronto, ON, Canada M3J 1P3 \\ Correspondence should be addressed to Chris I. Ardern; cardern@yorku.ca
}

Received 22 May 2015; Accepted 21 September 2015

Academic Editor: Aaron L. Sverdlov

Copyright (C) 2015 T. Kanagasabai and C. I. Ardern. This is an open access article distributed under the Creative Commons Attribution License, which permits unrestricted use, distribution, and reproduction in any medium, provided the original work is properly cited.

\begin{abstract}
Sleep is vital for cardiometabolic health, but a societal shift toward poor sleep is a prominent feature of many modern cultures. Concurrently, factors such as diet and lifestyle have also changed and may mediate the relationship between sleep quality and cardiometabolic health. Objectives were to explore (1) the interrelationship and (2) mediating effect of inflammation, oxidative stress, and antioxidants on sleep quality and cardiometabolic health. Cross-sectional data from the US National Health and Nutritional Examination Survey 2005-06 ( $\geq 20$ y; $N=2,072)$ was used. Cardiometabolic health was defined as per the Joint Interim Statement; overall sleep quality was determined from six sleep habits and categorized as good, fair, poor, and very poor. Fair quality sleepers had optimal inflammation, oxidative stress, and antioxidant levels. Inflammation was above the current clinical reference range across all sleep quality categories, while oxidative stress was only within the clinical reference range for fair sleep quality. Selected sleep quality-cardiometabolic health relationships were mediated by inflammation, oxidative stress, and antioxidants and were moderated by sex. Our results provide initial evidence of a potential role for inflammation, oxidative stress, and antioxidants in the pathway between poor sleep quality-cardiometabolic decline. Further prospective research is needed to confirm our results.
\end{abstract}

\section{Introduction}

Sleep is a vital human process needed for optimal immune, cardiometabolic, and cognitive health [1]. However, a societal trend toward less sleep and poorer quality sleep is a common feature in many developed countries $[2,3]$. Whether sleep duration or quality is more important for health remains unclear, but some research suggests they modify each other's associations with health [4]. After adjusting for sleep duration, significant associations between sleep quality and cardiometabolic health were found in several population studies [4-6].

However, few studies have explored the association of sleep quality with inflammation, oxidative stress, and antioxidants levels $[7,8]$. Observational studies support a modestto-strong correlation between sleep quality measures and inflammation (i.e., C-Reactive Protein (CRP)) $[9,10]$, but the relationship may be sex-specific. For instance, Liu et al. [10] found that poor quality sleep was significantly associated with CRP in women, but not men. Additionally, the role of oxidative stress in sleep quality as well as the beneficial effect of antioxidant therapy has been demonstrated in obstructive sleep apnea patients [11]. Furthermore, the relationship between these factors (i.e., inflammation, oxidative stress, and antioxidants) and cardiometabolic health is also known and collectively suggests that abnormal levels of inflammation, oxidative stress, and insufficient antioxidants are associated with many age-related diseases, including diabetes, cardiovascular disease, and cancer [12]. Previously, we explored the interrelationship and mediating effect of inflammation (i.e., CRP), oxidative stress (i.e., $\gamma$-glutamyl transferase (GGT)), and antioxidants (i.e., bilirubin, carotenoids, uric acid, and vitamins A, C, D, and E) on sleep duration and cardiometabolic health [13]. However, the role of sleep quality within this context has not yet been explored. Thus, the purpose of this study is to (i) explore the interrelationship 
between sleep quality and inflammation (CRP) [14], oxidative stress (GGT) [15], and antioxidants (bilirubin, carotenoids, uric acid, and vitamins A, C, D, and E) [16, 17] and (ii) quantify the indirect mediating effect of these factors on the sleep quality-cardiometabolic health relationships in freeliving adults.

\section{Methods}

2.1. Participants. Data for this analysis was drawn from the US National Health and Nutrition Examination Survey (NHANES) [20]. NHANES is a series of nationally representative cross-sectional studies designed to assess the health and nutritional status of the US noninstitutionalized civilian population of all ages and ethnicities. Approximately 10,000 persons are sampled biannually, and data are collected from personal interviews, standardized physical examinations, and laboratory samples. NHANES 2005-2006 cycle, which had an initial sample of 10,348 individuals, was used in this study. Subsequent exclusions were made for age $(<20$ y: $N=$ $5,369)$, pregnancy $(N=336)$, missing MetS components $\left(N_{\text {waist circumference }}=455, N_{\text {triglyceride }}=196, N_{\text {blood pressure }}=\right.$ $79, N_{\text {fasting plasma glucose }}=1,826$, and $\left.N_{\text {HDL cholesterol }}=0\right)$, and missing sleep quality variables $(N=15)$ for a final analytic sample of 2,072 .

2.2. Metabolic Syndrome and Cardiometabolic Health. The Joint Interim Statement was used to define metabolic syndrome (MetS) [ $\geq 3$ of elevated waist circumference: men $(\geq 102 \mathrm{~cm})$ and women $(\geq 88 \mathrm{~cm})$; elevated triglycerides or medication: $\geq 1.69 \mathrm{mM}$; low HDL cholesterol or medication: men $(<1.04 \mathrm{mM})$ and women $(<1.29 \mathrm{mM})$; elevated blood pressure or medication: systolic $(\geq 130 \mathrm{mmHg})$ and/or diastolic ( $\geq 85 \mathrm{mmHg}$ ); and elevated fasting plasma glucose or medication use $(\geq 5.6 \mathrm{mM})]$ [21]. These criteria were summed to determine the number of MetS components $[0,1,2,3,4,5]$. Cardiometabolic health markers assessed were the individual MetS components included in the Joint Interim Statement.

2.3. Sleep Quality. The Sleep Disorders Questionnaire was administered to participants aged $\geq 16$ y (limited to adults $\geq 20$ y for our analysis), who reported their typical sleep habits for the past month [20]. This questionnaire contains items from two previously validated sleep questionnaires [22]. Overall sleep quality was determined from six questions: "How often did you have trouble falling asleep?"; "How often did you wake up during night and had trouble getting back to sleep?"; "How often did you wake up too early in morning and were unable to get back to sleep?"; "How often did you feel unrested during the day, no matter how many hours of sleep you had?"; "How often did you feel excessively or overly sleepy during day?"; and, "How often did you not get enough sleep?" Participants' responses to each question [0 = never; $1=$ rarely (1 time a month); $2=$ sometimes $(2-4$ times a month); 3 = often (5-15 times a month); and $4=$ almost always (16-30 times a month)] were summed to obtain an index of overall sleep quality. The sleep quality score was subsequently categorized as good $(0$ to $<3)$; fair $(3$ to $<7)$; poor $(7$ to $<12)$; and very poor ( $\geq 12$ to 24$)$ [3].
2.4. Mediators and Population Descriptors. Mediators considered in this study were CRP, GGT, bilirubin, carotenoids, uric acid, and vitamins A, C, D, and E and were obtained from laboratory files [23]. Blood samples were collected by certified phlebotomists, and we used the morning session's data which contained blood samples after an overnight fast [23]. Demographic variables used to describe the population include age (categorized as 20 to $<40 \mathrm{y}, 40$ to $<65 \mathrm{y}$, and $\geq 65 \mathrm{y}$ ), sex (men, women), ethnicity (Non-Hispanic White, Non-Hispanic Black, Mexican American, and Others), income $(<\$ 20,000, \$ 20,000-44,999$, and $\geq \$ 45,000)$, education ( $<$ high school, high school, and college), alcohol intake $(0,<3$, and $\geq 3$ drinks per day), smoking history [current (if smoking now), past (if smoked $\geq 100$ cigarettes in one's life but not current smoker), or never (if smoked $<100$ cigarettes in one's life)], and recreational physical activity (PA) adherence (none reported, <500 metabolic equivalent $(\mathrm{MET}) \cdot \mathrm{min} / \mathrm{wk}$ and $\geq 500 \mathrm{MET} \cdot \mathrm{min} / \mathrm{wk})$ [24, 25]. To estimate MET.min/wk for recreational PA adherence, we used NHANES suggested MET values, which were then categorized according to the PA guidelines for Americans [26].

2.5. Statistics. Mean and $95 \%$ confidence interval (CI) for continuous variables and frequency (\%) and 95\% CI for categorical variables were determined for each descriptor, by category of sleep quality. ANOVA (with post hoc Tukey's test) and $\chi^{2}$ tests were used, as appropriate, to test for any differences in demographic and behavioral characteristics of sleep quality groups. The interrelationship between sleep quality and CRP, GGT, bilirubin, carotenoids, uric acid, and vitamins $\mathrm{A}, \mathrm{C}, \mathrm{D}$, and $\mathrm{E}$ was determined and presented visually relative to the American Medical Association's clinical reference ranges [19]. Subsequent to this, the indirect mediation effect of CRP, GGT, bilirubin, carotenoids, uric acid, and vitamins $\mathrm{A}, \mathrm{C}, \mathrm{D}$, and $\mathrm{E}$ on the sleep-cardiometabolic health relationship was estimated using (i) logistic regression for binary outcomes (i.e., MetS) and (ii) general linear models for the number of MetS components and individual cardiometabolic parameters [18].

Through a series of regression analyses, indirect mediation helps explain the underlying relationship between an exposure and an outcome variable through a third (mediatory) variable [18] (Figure 1). These relationships are depicted by four pathways: regression between exposure and mediator (path $a$ ); regression between mediator and outcome while adjusting for the exposure (path $b$ ); regression between exposure and outcome (path $c$ ); and regression between exposure and outcome while adjusting for the mediator (path $\left.c^{\prime}\right)$ [18]. Participants with a missing mediator variable were excluded from each regression analysis to ensure the products of $a b$ and $c-c^{\prime}$ were equivalent [18]. The product of $\mathrm{ab}$ was subsequently used to classify indirect effects as large $(\geq 0.25)$, moderate $(\geq 0.09)$, modest $(\geq 0.01)$, and weak $(<0.01)$ [18]. To detect a moderate indirect effect with $80 \%$ power, $n=$ 105 participants were needed in each sleep quality category. Significance for indirect effect was tested with the Sobel and Joint Significance tests.

To ensure the representativeness of the data, the medical exam sample weight from the demographics data file was 


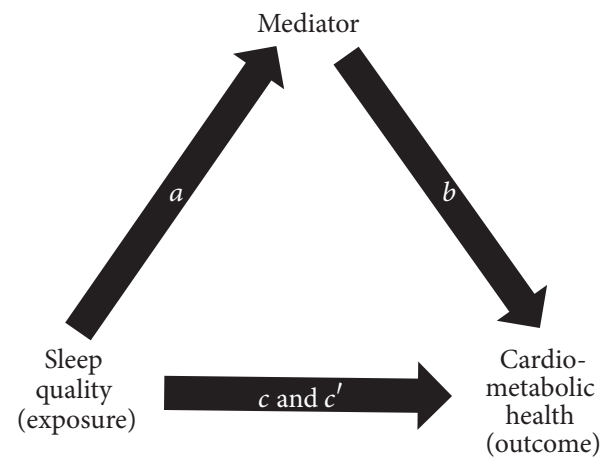

FIGURE 1: Multiple regression method of the indirect mediation model [18]. Path $a$ indicates the path from sleep quality (exposure) to mediator (i.e., inflammation, oxidative stress, and antioxidant). Path $b$ indicates the path from mediator to outcome (i.e., metabolic syndrome (MetS), number of MetS components, and individual MetS components) controlling for the mediator. Path $c$ indicates the path from exposure to outcome. Path $c^{\prime}$ indicates the path from exposure to outcome controlling for the mediator.

used to weight all analyses [20]. All analyses were conducted in SAS v9.3 (Cary, NC, U.S.A) with statistical significance set at an $\alpha$ of 0.05 .

\section{Results}

Table 1 describes the characteristics of the US adult population, stratified by sleep quality categories. In general, sleep quality varied by sex, age, ethnicity, education, recreational PA, and smoking. Specifically, middle aged adults (40 to $<65$ y) tended to have very poor quality sleep, while older adults reported good quality sleep compared to other sleep quality categories. Very poor sleep quality was also more common among women, Non-Hispanic Whites, and current smokers. Compared to good and very poor quality sleepers, both fair and poor sleepers attained more recreational PA, half of whom, approximately, met the PA guidelines for Americans.

Figure 2 illustrates the interrelationship between sleep quality and inflammation (a), oxidative stress (b), and antioxidants (c)-(i) with clinical reference ranges shaded in gray. Those reporting fair, but not good, quality of sleep had the optimal inflammation and oxidative stress profiles. However, inflammation was above the clinical reference range across all sleep quality categories. For GGT, all levels of sleep quality except those with very poor quality sleep had mean values within the clinical reference range. All antioxidants were within the clinical reference range and were generally optimal among fair or poor quality sleepers. CRP and vitamin C levels were statistically different for fair versus very poor quality sleep.

Overall, vitamins $\mathrm{A}$ and $\mathrm{C}$ were modest mediators of the sleep quality-MetS and sleep quality-number of MetS components relationships (Table 2). Further exploration of the indirect effect revealed these antioxidants were also moderate $(\geq 0.09)$ mediators of the sleep quality-waist circumference and sleep quality-systolic blood pressure relationships
(Table 3). Additionally, the sleep quality-waist circumference and sleep quality-diastolic blood pressure relationships were moderately mediated by CRP.

While some minor differences between men and women were observed for the sleep quality-MetS and sleep qualitynumber of MetS components relationships (men: Table 4; women: Table 5), the differences were larger for some individual components (men: Table 6; women: Table 7). In women, $\mathrm{CRP}$, uric acid, and vitamin $\mathrm{C}$ were large mediators of the sleep quality-waist circumference relationship and moderately mediated by carotenoids and GGT. Uric acid was also a large mediator of the sleep quality-systolic blood pressure relationship in women, while CRP and carotenoids were moderate mediators. Finally, vitamin $\mathrm{C}$ was a moderate mediator of the sleep quality-diastolic blood pressure relationship in women.

\section{Discussion}

We found that very poor sleep quality was more common amongst women, Non-Hispanic Whites, current smokers, and middle aged adults ( 40 to $<65 \mathrm{y}$ ), whereas older adults were more likely to report good quality sleep. Optimal inflammation and oxidative stress profiles were found amongst fair quality sleepers, while some antioxidants were also optimal amongst fair and poor quality sleepers. While most sleep quality-MetS or sleep quality-number of MetS components relationship were not significant, selected sleep qualitycardiometabolic health relationships were moderately mediated by CPR and vitamins A and C. Additionally, in women only, the indirect effects were moderate-to-large for CRP, GGT, carotenoids, uric acid, and vitamin C.

4.1. Inflammation, Oxidative Stress, and Antioxidants Profiles. Our finding of optimal inflammation, oxidative stress, and antioxidant levels amongst the fair or poor sleep quality categories was surprising, as we expected optimal levels amongst those with at least good sleep quality. To date, relatively few studies have investigated the interrelationship between inflammation, oxidative stress, and antioxidants with sleep quality $[7,8,27-29]$. In one such study $(n=$ 24), adherence to a kiwi diet (2 kiwi/night for 4 weeks), a fruit rich in vitamins $\mathrm{C}$ and $\mathrm{E}$ and serotonin, improved sleep onset and duration [27]. Another similar study ( $n=$ 20) found that tart cherry juice, rich in vitamins $A$ and $C$, improved sleep quality, suggesting increased melatonin levels as a possible mechanism for this effect [28]. However, neither of these studies measured serum antioxidant levels [27, 28]. In sleep disordered populations, elevated levels of inflammation and oxidative stress are common features [29]; and overall antioxidant capacity tends to decrease with age [30].

Another possible explanation is the definition of sleep quality, which is based on the six questions that were assigned equal weights [3]. In a previous analysis, we found that the odds of MetS varied between questions, with no significant relationship for getting enough sleep (OR:1.06) and roughly twofold increases for trouble falling asleep, feeling overly sleepy, and waking up during the night, after adjusting for 
TABLE 1: Characteristics of the US adult population $\geq 20$ years of age.

\begin{tabular}{|c|c|c|c|c|c|}
\hline \multirow{2}{*}{ Characteristics } & \multicolumn{4}{|c|}{ Sleep quality } & \multirow{2}{*}{$p$ valu } \\
\hline & Good $(n=428)$ & Fair $(n=488)$ & Poor $(n=579)$ & Very poor $(n=577)$ & \\
\hline Age (mean (95\% CI)) & $51.5(48.7,54.3)$ & $48.9(46.2,51.6)$ & $46.6(44.4,48.8)$ & $46.7(44.5,48.9)$ & $<0.05$ \\
\hline \multicolumn{6}{|c|}{ Age categories (\% $(95 \% \mathrm{CI}))$} \\
\hline 20 to $<40$ years & $28.2(21.7,34.7)$ & $33.9(28.6,39.2)$ & $36.8(31.8,41.8)$ & $33.7(29.1,38.4)$ & \multirow{3}{*}{$<0.05$} \\
\hline 40 to $<65$ years & $45.9(40.4,51.5)$ & $44.8(39.2,50.4)$ & $47.1(44.1,50.1)$ & $51.3(45.7,56.9)$ & \\
\hline$\geq 65$ years & $25.9(20.3,31.4)$ & $21.3(15.9,26.6)$ & $16.1(12.2,20.0)$ & $15.0(10.0,19.9)$ & \\
\hline \multicolumn{6}{|l|}{ Sex } \\
\hline Men & $60.8(55,66.6)$ & $51.5(46.5,56.4)$ & $51.1(47.5,54.8)$ & $43.4(39.5,47.4)$ & \multirow{2}{*}{$<0.05$} \\
\hline Women & $39.2(33.4,45.0)$ & $48.5(43.6,53.5)$ & $48.9(45.2,52.5)$ & $56.6(52.6,60.5)$ & \\
\hline \multicolumn{6}{|l|}{ Ethnicity } \\
\hline Non-Hispanic White & $58.1(49.1,67.2)$ & $73.8(66.7,80.9)$ & $75.2(67.4,82.9)$ & $73.6(68.3,78.9)$ & \multirow{4}{*}{$<0.05$} \\
\hline Non-Hispanic Black & $15.6(10.7,20.5)$ & $10.0(5.5,14.6)$ & $8.6(5.1,12.2)$ & $13.0(8.9,17.1)$ & \\
\hline Mexican American & $14.8(9.1,20.6)$ & $7.0(3.5,10.6)$ & $6.6(4.6,8.6)$ & $5.3(3.2,7.3)$ & \\
\hline Others & $11.4(6.0,16.8)$ & $9.2(3.6,14.7)$ & $9.6(5.1,14.1)$ & $8.1(5.0,11.3)$ & \\
\hline \multicolumn{6}{|l|}{ Education } \\
\hline$<$ High school & $26.1(18.7,33.5)$ & $16.5(11.2,21.8)$ & $14.2(10.9,17.5)$ & $16.8(12.5,21.1)$ & \multirow{3}{*}{$<0.05$} \\
\hline High school & $25.5(19.0,32.1)$ & $24.9(20.1,29.7)$ & $24.4(20.2,28.6)$ & $27.7(24.0,31.4)$ & \\
\hline College & $48.4(36.2,60.6)$ & $58.6(51.5,65.7)$ & $61.4(56.0,66.8)$ & $55.5(50.0,61.0)$ & \\
\hline \multicolumn{6}{|l|}{ Income } \\
\hline$<\$ 20,000$ & $16.8(12.0,21.6)$ & $14.7(10.8,18.6)$ & $12.9(9.2,16.7)$ & $18.8(14.1,23.6)$ & \multirow{3}{*}{ NS } \\
\hline$\$ 20,000-44,999$ & $33.7(25.8,41.5)$ & $33.1(27.2,39.0)$ & $30.3(23.6,37.0)$ & $31.5(24.0,39.0)$ & \\
\hline$\geq \$ 45,000$ & $49.5(39.5,59.6)$ & $52.2(45.6,58.9)$ & $56.8(49,64.5)$ & $49.7(42.1,57.3)$ & \\
\hline \multicolumn{6}{|l|}{ Smoking } \\
\hline None & $52.1(43.0,61.3)$ & $47.7(40.8,54.5)$ & $52.9(47.9,58)$ & $42.8(36.6,49.0)$ & \multirow{3}{*}{$<0.05$} \\
\hline Current & $20.7(14.7,26.8)$ & $20.6(14.9,26.4)$ & $22.1(17.1,27.2)$ & $34.3(29.7,39.0)$ & \\
\hline Past & $27.1(21.5,32.8)$ & $31.7(26.8,36.6)$ & $24.9(20.8,29.0)$ & $22.8(19.3,26.4)$ & \\
\hline \multicolumn{6}{|l|}{ Alcohol intake } \\
\hline 0 drinks/d & $36.6(30.9,42.3)$ & $29.8(23.3,36.3)$ & $28.1(22.2,34.0)$ & $33.6(29.4,37.8)$ & \multirow{3}{*}{ NS } \\
\hline$<3$ drinks/d & $41.3(34.4,48.3)$ & $46.3(39.5,53.1)$ & $44.8(39.0,50.5)$ & $39.7(34.5,44.8)$ & \\
\hline$\geq 3$ drinks/d & $22.1(16.9,27.3)$ & $23.9(19.2,28.6)$ & $27.1(22.4,31.9)$ & $26.8(21.8,31.7)$ & \\
\hline \multicolumn{6}{|c|}{ Recreational physical activity } \\
\hline None reported & $41.2(34.2,48.1)$ & $28.2(24.2,32.3)$ & $28.2(22.9,33.5)$ & $35.6(31.2,39.9)$ & \multirow{3}{*}{$<0.05$} \\
\hline$<500 \mathrm{MET} \cdot \mathrm{min} / \mathrm{w}$ & $16.4(11.3,21.5)$ & $22.2(17.8,26.6)$ & $25.1(20.2,30.1)$ & $22.4(18.3,26.5)$ & \\
\hline$\geq 500 \mathrm{MET} \cdot \mathrm{min} / \mathrm{w}$ & $42.4(36.9,48.0)$ & $49.6(44.1,55.1)$ & $46.6(41.9,51.4)$ & $42.1(35.2,49.0)$ & \\
\hline
\end{tabular}

Mean $(95 \%$ CI) for continuous variables and \% $(95 \%$ CI) for categorical variables. Overall sleep quality was calculated based on six questions on sleep habits of participants and categorized into quartiles: good ( $<3)$, fair ( $\geq 3$ to 7$)$, poor $(\geq 7$ to 12$)$, and very poor $(\geq 12) . p<0.05$, two-sided; ANOVA or Chi-square, as appropriate. NS: not significant. Sum of weights $=95,276,598$.

age, sex, ethnicity, education, income, smoking, recreational physical activity, and sleep duration (unpublished data). Therefore, future work may benefit from the development of a recalibrated (weighted index) of sleep quality.

4.2. Indirect Mediation Effect. Evaluating the indirect effect of inflammation, oxidative stress, and antioxidants on the relationship between sleep quality and cardiometabolic health was the second aim of our study. Although our overall findings were weak or modest for the sleep quality-MetS and sleep quality-number of MetS components relationships, moderate indirect effects by CPR and vitamin C on the sleep quality-waist circumference and vitamin A for sleep qualitysystolic blood pressure relationships warrant discussion.
4.2.1. Waist Circumference. Although the relationship between poor sleep quality and body weight is well known [31], little work has been done to examine the association with sleep quality. Positive associations between waist circumference and CRP or vitamin $\mathrm{C}$ have nonetheless been found $[32,33]$. Evidence also suggests that sleep architecture may be altered in individuals with obesity; that is, rapid eye movement stage of sleep may occur earlier compared to normal weight individuals [34]. In sleep disordered populations, the prevalence of obesity is high; and both sleep quality and obesity have been independently linked to increased inflammation and decreased antioxidants [7, 14, 31]. For instance, a large Finnish study reported that CRP was elevated $(\geq 9.52 \mathrm{nM})$ among men reporting frequent sleep 


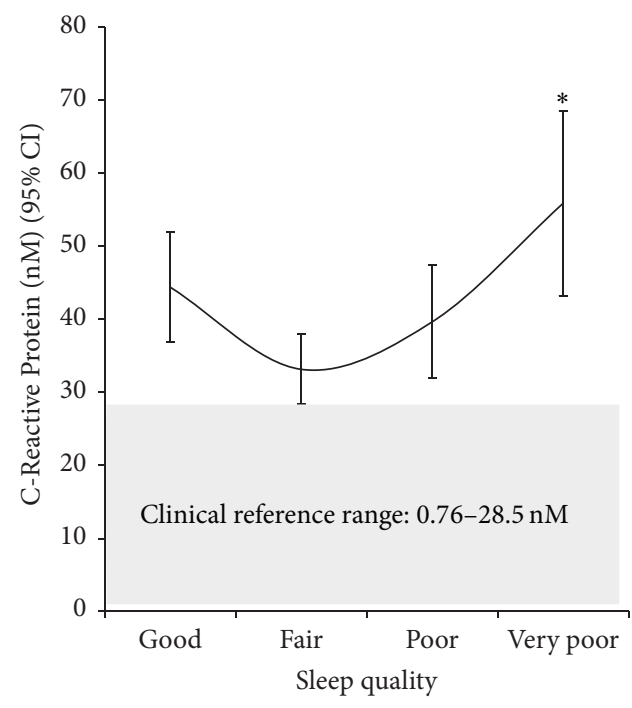

(a)

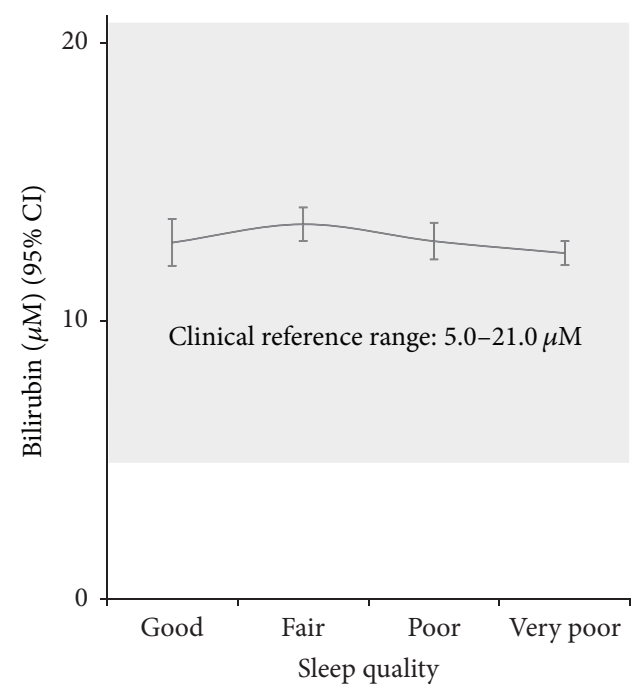

(c)

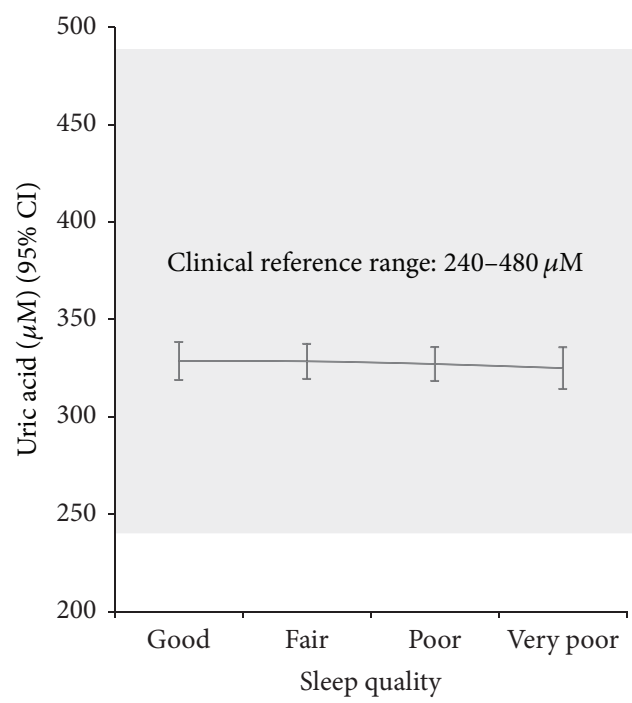

(e)

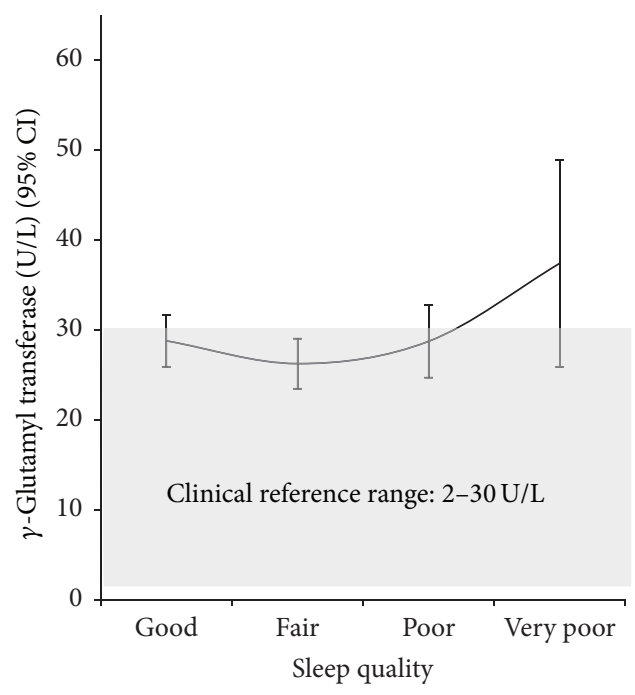

(b)

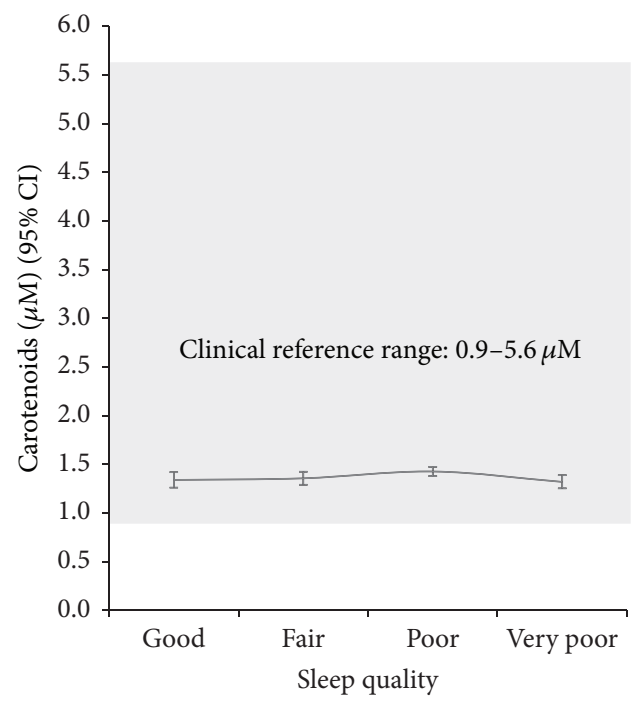

(d)

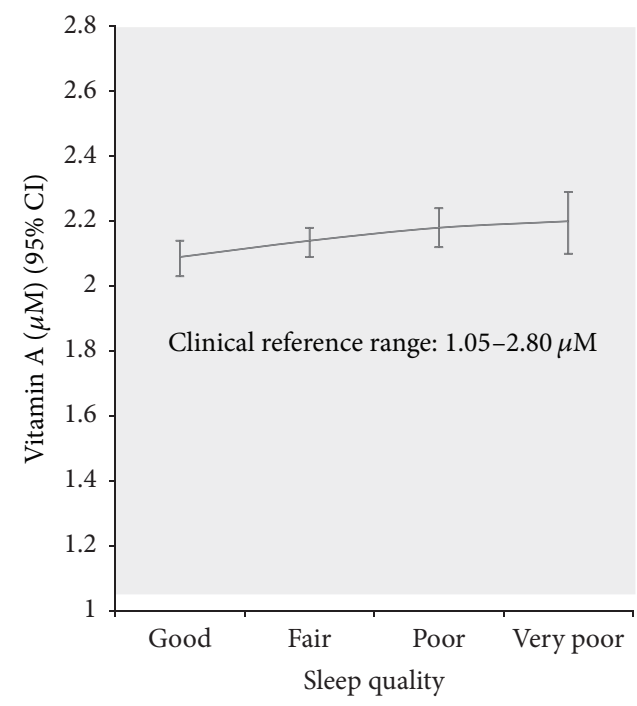

(f)

Figure 2: Continued. 


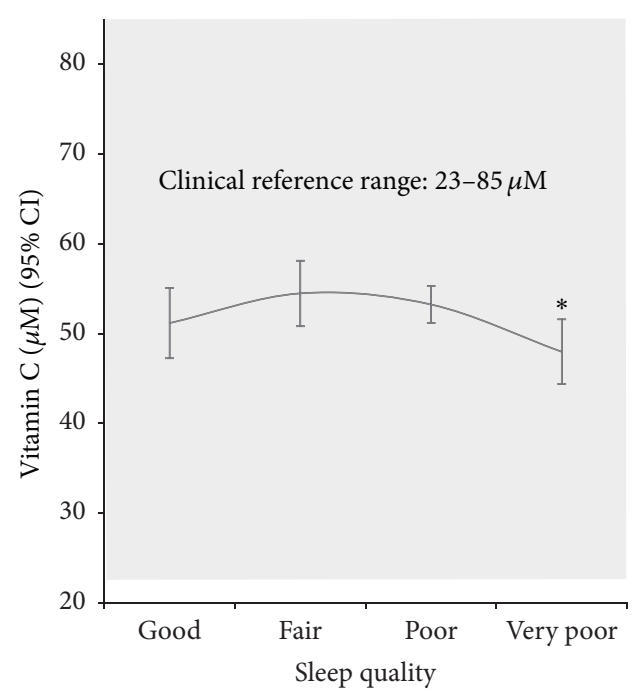

(g)

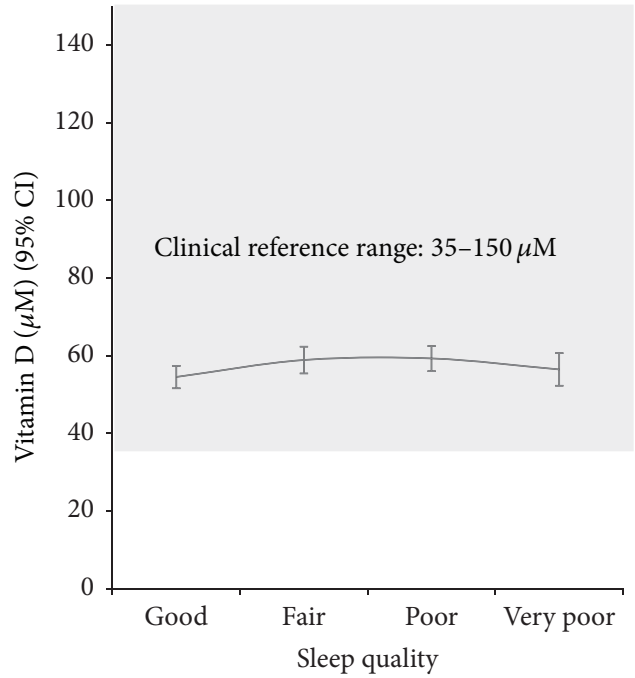

(h)

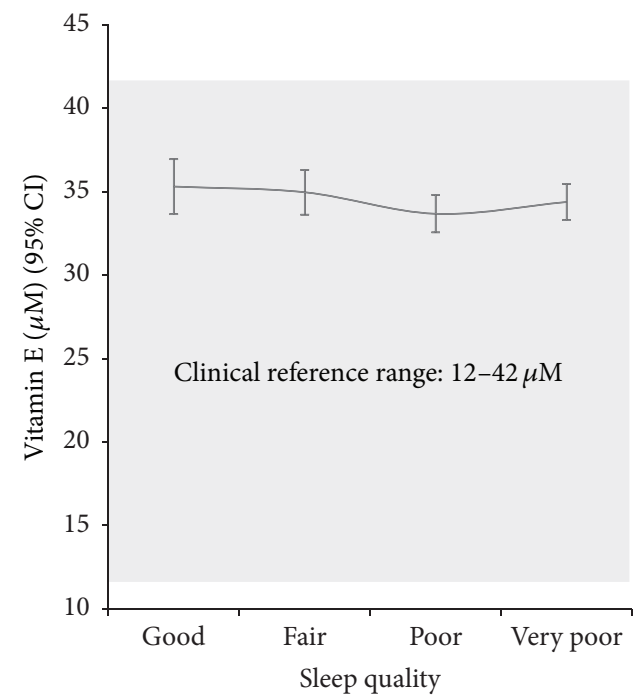

(i)

FIGURE 2: Interrelationship between sleep quality and inflammation (a), oxidative stress (b), and antioxidants ((c)-(i)). Gray shaded areas are clinical reference ranges [19]. ${ }^{*} p<0.05$ versus fair sleep quality.

TABLE 2: Indirect effect of mediators on the sleep quality-metabolic syndrome and sleep quality-number of MetS components relationship.

\begin{tabular}{|c|c|c|c|}
\hline \multirow{2}{*}{ Mediator } & \multirow{2}{*}{$\begin{array}{c}c-c^{\prime} \\
\text { MetS }\end{array}$} & \multicolumn{2}{|c|}{$a b\left(95 \% \mathrm{CI}_{\text {sobel }}\right)$} \\
\hline & & MetS & Number of MetS components \\
\hline C-Reactive Protein (nM) & 0.012 & $0.014(-0.007,0.035)$ & $0.011(-0.004,0.026)$ \\
\hline$\gamma$-Glutamyl transferase $(\mathrm{U} / \mathrm{L})$ & 0.022 & $0.042(-0.006,0.090)$ & $0.011(-0.003,0.025)$ \\
\hline Bilirubin $(\mu \mathrm{M})$ & 0.006 & $0.006(-0.001,0.013)$ & $0.006(-0.001,0.012)$ \\
\hline Carotenoids $(\mu \mathrm{M})$ & 0.002 & $0.002(-0.012,0.016)$ & $0.001(-0.010,0.013)$ \\
\hline Uric acid $(\mu \mathrm{M})$ & -0.009 & $-0.011(-0.038,0.017)$ & $-0.008(-0.030,0.013)$ \\
\hline Vitamin $\mathrm{A}(\mu \mathrm{M})$ & 0.017 & $0.016(0.001,0.031)^{*}$ & $0.015(0.001,0.028)^{*}$ \\
\hline Vitamin $\mathrm{C}(\mu \mathrm{M})$ & 0.019 & $0.018(0.003,0.034)^{*}$ & $0.014(0.002,0.026)^{*}$ \\
\hline Vitamin D (nM) & -0.004 & $-0.005(-0.024,0.014)$ & $-0.004(-0.021,0.013)$ \\
\hline Vitamin $\mathrm{E}(\mu \mathrm{M})$ & -0.015 & $-0.016(-0.040,0.008)$ & $-0.014(-0.035,0.006)$ \\
\hline
\end{tabular}

MetS is metabolic syndrome. $c-c^{\prime}$ and $a b$ are indirect effect. ${ }^{*}$ Significant for Joint test, and bold means significant for Sobel test. 
TABLE 3: Indirect effect of mediators on the sleep quality-individual MetS component relationship.

\begin{tabular}{|c|c|c|c|c|c|c|}
\hline \multirow{2}{*}{ Mediator } & \multicolumn{6}{|c|}{$a b\left(95 \% \mathrm{CI}_{\text {sobel }}\right)$} \\
\hline & $\begin{array}{c}\text { Waist } \\
\text { circumference }\end{array}$ & $\begin{array}{l}\text { Systolic blood } \\
\text { pressure }\end{array}$ & $\begin{array}{l}\text { Diastolic blood } \\
\text { pressure }\end{array}$ & Triglycerides & HDL cholesterol & $\begin{array}{c}\text { Fasting plasma } \\
\text { glucose }\end{array}$ \\
\hline $\begin{array}{l}\text { C-Reactive Protein } \\
\text { (nM) }\end{array}$ & $\begin{array}{c}0.204 \\
(-0.004,0.412)^{*}\end{array}$ & $\begin{array}{c}0.058 \\
(-0.023,0.140)\end{array}$ & $\begin{array}{c}0.035 \\
(-0.009,0.078)^{*}\end{array}$ & $\begin{array}{c}0.005 \\
(-0.002,0.011)\end{array}$ & $\begin{array}{c}-0.001 \\
(-0.003,0.000)\end{array}$ & $\begin{array}{c}0.009 \\
(-0.002,0.020)\end{array}$ \\
\hline $\begin{array}{l}\gamma \text {-Glutamyl } \\
\text { transferase }(\mathrm{U} / \mathrm{L})\end{array}$ & $\begin{array}{c}0.101 \\
(-0.025,0.226)\end{array}$ & $\begin{array}{c}0.101 \\
(-0.037,0.240)\end{array}$ & $\begin{array}{c}0.049 \\
(-0.013,0.112)\end{array}$ & $\begin{array}{c}0.010 \\
(-0.004,0.023)\end{array}$ & $\begin{array}{c}-0.001 \\
(-0.002,0.001)\end{array}$ & $\begin{array}{c}0.013 \\
(-0.005,0.032)\end{array}$ \\
\hline Bilirubin $(\mu \mathrm{M})$ & $\begin{array}{c}0.016 \\
(-0.110,0.142)\end{array}$ & $\begin{array}{c}0.014 \\
(-0.06,0.088)\end{array}$ & $\begin{array}{c}-0.002 \\
(-0.012,0.008)\end{array}$ & $\begin{array}{c}0.000 \\
(0.000,0.000)\end{array}$ & $\begin{array}{c}0.000 \\
(-0.003,0.002)\end{array}$ & $\begin{array}{c}0.000 \\
(-0.008,0.007)\end{array}$ \\
\hline Carotenoids $(\mu \mathrm{M})$ & $\begin{array}{c}0.094 \\
(-0.023,0.211)\end{array}$ & $\begin{array}{c}0.071 \\
(-0.01,0.151)\end{array}$ & $\begin{array}{c}-0.010 \\
(-0.034,0.014)\end{array}$ & $\begin{array}{c}0.001 \\
(-0.001,0.004)\end{array}$ & $\begin{array}{c}-0.001 \\
(-0.003,0.000)\end{array}$ & $\begin{array}{c}0.004 \\
(-0.004,0.012)\end{array}$ \\
\hline Uric acid $(\mu \mathrm{M})$ & $\begin{array}{c}-0.115 \\
(-0.411,0.181)\end{array}$ & $\begin{array}{c}-0.051 \\
(-0.192,0.09)\end{array}$ & $\begin{array}{c}-0.013 \\
(-0.052,0.027)\end{array}$ & $\begin{array}{c}-0.004 \\
(-0.016,0.007)\end{array}$ & $\begin{array}{c}0.002 \\
(-0.003,0.006)\end{array}$ & $\begin{array}{c}0.000 \\
(-0.003,0.002)\end{array}$ \\
\hline Vitamin $\mathrm{A}(\mu \mathrm{M})$ & $\begin{array}{c}0.046 \\
(-0.022,0.115)\end{array}$ & $\begin{array}{c}0.158 \\
(0.023,0.294)^{*}\end{array}$ & $\begin{array}{c}0.001 \\
(-0.034,0.037)\end{array}$ & $\begin{array}{c}0.019 \\
(0.002,0.036)^{*}\end{array}$ & $\begin{array}{c}0.001 \\
(-0.001,0.002)\end{array}$ & $\begin{array}{c}0.000 \\
(-0.005,0.006)\end{array}$ \\
\hline Vitamin $\mathrm{C}(\mu \mathrm{M})$ & $\begin{array}{c}0.227 \\
(0.039,0.414)^{*}\end{array}$ & $\begin{array}{c}0.049 \\
(-0.028,0.125)\end{array}$ & $\begin{array}{c}0.076 \\
(0.008,0.145)^{*}\end{array}$ & $\begin{array}{c}0.010 \\
(0.001,0.020)^{*}\end{array}$ & $\begin{array}{c}-0.005 \\
(-0.009,-0.001)^{*}\end{array}$ & $\begin{array}{c}0.011 \\
(0.003,0.018)^{*}\end{array}$ \\
\hline Vitamin D (nM) & $\begin{array}{c}-0.057 \\
(-0.292,0.178)\end{array}$ & $\begin{array}{c}-0.034 \\
(-0.143,0.074)\end{array}$ & $\begin{array}{c}-0.008 \\
(-0.040,0.023)\end{array}$ & $\begin{array}{c}-0.002 \\
(-0.008,0.005)\end{array}$ & $\begin{array}{c}0.001 \\
(-0.003,0.004)\end{array}$ & $\begin{array}{c}-0.004 \\
(-0.018,0.011)\end{array}$ \\
\hline Vitamin $\mathrm{E}(\mu \mathrm{M})$ & $\begin{array}{c}-0.064 \\
(-0.160,0.032)\end{array}$ & $\begin{array}{c}-0.099 \\
(-0.266,0.068)\end{array}$ & $\begin{array}{c}-0.035 \\
(-0.092,0.022)\end{array}$ & $\begin{array}{c}-0.027 \\
(-0.067,0.012)\end{array}$ & $\begin{array}{c}0.000 \\
(-0.001,0.001)\end{array}$ & $\begin{array}{c}-0.010 \\
(-0.025,0.005)\end{array}$ \\
\hline
\end{tabular}

$a b$ is indirect effect. ${ }^{*}$ Significant for Joint test, and bold means significant for Sobel test.

TABLE 4: Indirect effect of mediators on the sleep quality-metabolic syndrome and sleep quality-number of MetS components relationship for men.

\begin{tabular}{|c|c|c|c|}
\hline \multirow{2}{*}{ Mediator } & \multirow{2}{*}{$\begin{array}{c}c-c^{\prime} \\
\text { MetS }\end{array}$} & \multicolumn{2}{|c|}{$a b\left(95 \% \mathrm{CI}_{\text {sobel }}\right)$} \\
\hline & & MetS & Number of MetS components \\
\hline C-Reactive Protein (nM) & 0.002 & $0.001(-0.006,0.008)$ & $0.002(-0.006,0.010)$ \\
\hline$\gamma$-Glutamyl transferase $(\mathrm{U} / \mathrm{L})$ & 0.024 & $0.042(-0.013,0.097)$ & $0.014(-0.003,0.031)$ \\
\hline Bilirubin $(\mu \mathrm{M})$ & 0.001 & $0.000(-0.013,0.013)$ & $0.000(-0.011,0.011)$ \\
\hline Carotenoids $(\mu \mathrm{M})$ & 0.010 & $-0.014(-0.032,0.004)$ & $-0.011(-0.024,0.002)$ \\
\hline Uric acid $(\mu \mathrm{M})$ & 0.013 & $-0.001(-0.06,0.058)$ & $-0.001(-0.041,0.039)$ \\
\hline Vitamin $\mathrm{A}(\mu \mathrm{M})$ & 0.017 & $0.015(-0.004,0.034)$ & $0.014(0.000,0.028)^{*}$ \\
\hline Vitamin $C(\mu \mathrm{M})$ & 0.012 & $0.007(-0.021,0.036)$ & $0.005(-0.016,0.026)$ \\
\hline Vitamin D (nM) & -0.009 & $-0.010(-0.024,0.004)$ & $-0.011(-0.024,0.002)$ \\
\hline Vitamin $\mathrm{E}(\mu \mathrm{M})$ & -0.018 & $-0.029(-0.057,0.000)^{*}$ & $-0.026(-0.050,-0.002)^{*}$ \\
\hline
\end{tabular}

MetS is metabolic syndrome. $a b$ is indirect effect. * Significant for Joint test, and bold means significant for Sobel test.

TABLE 5: Indirect effect of mediators on the sleep quality-metabolic syndrome and sleep quality-number of MetS components relationship for women.

\begin{tabular}{|c|c|c|c|}
\hline \multirow{2}{*}{ Mediators } & \multirow{2}{*}{$\begin{array}{l}c-c^{\prime} \\
\text { MetS }\end{array}$} & \multicolumn{2}{|c|}{$a b\left(95 \% \mathrm{CI}_{\text {sobel }}\right)$} \\
\hline & & MetS & Number of MetS components \\
\hline C-Reactive Protein (nM) & 0.036 & $0.044(0.011,0.078)^{*}$ & $0.035(0.012,0.059)^{*}$ \\
\hline$\gamma$-Glutamyl transferase $(\mathrm{U} / \mathrm{L})$ & 0.042 & $0.049(-0.012,0.109)^{*}$ & $0.017(-0.001,0.035)^{*}$ \\
\hline Bilirubin $(\mu \mathrm{M})$ & 0.003 & $0.003(-0.005,0.011)$ & $0.005(-0.005,0.015)$ \\
\hline Carotenoids $(\mu \mathrm{M})$ & 0.012 & $0.020(0.000,0.040)$ & $0.017(0.000,0.033)$ \\
\hline Uric acid $(\mu \mathrm{M})$ & 0.035 & $0.056(0.007,0.105)^{*}$ & $0.043(0.005,0.08)^{*}$ \\
\hline Vitamin $\mathrm{A}(\mu \mathrm{M})$ & 0.022 & $0.025(-0.006,0.055)$ & $0.021(-0.005,0.047)$ \\
\hline Vitamin $C(\mu \mathrm{M})$ & 0.031 & $0.032(0.008,0.055)^{*}$ & $0.022(0.004,0.041)^{*}$ \\
\hline Vitamin D (nM) & 0.006 & $0.013(-0.036,0.062)$ & $0.010(-0.028,0.048)$ \\
\hline Vitamin $\mathrm{E}(\mu \mathrm{M})$ & -0.014 & $-0.032(-0.062,-0.002)^{*}$ & $-0.003(-0.030,0.024)$ \\
\hline
\end{tabular}

MetS is metabolic syndrome. $a b$ is indirect effect. * Significant for Joint test, and bold means significant for Sobel test. 


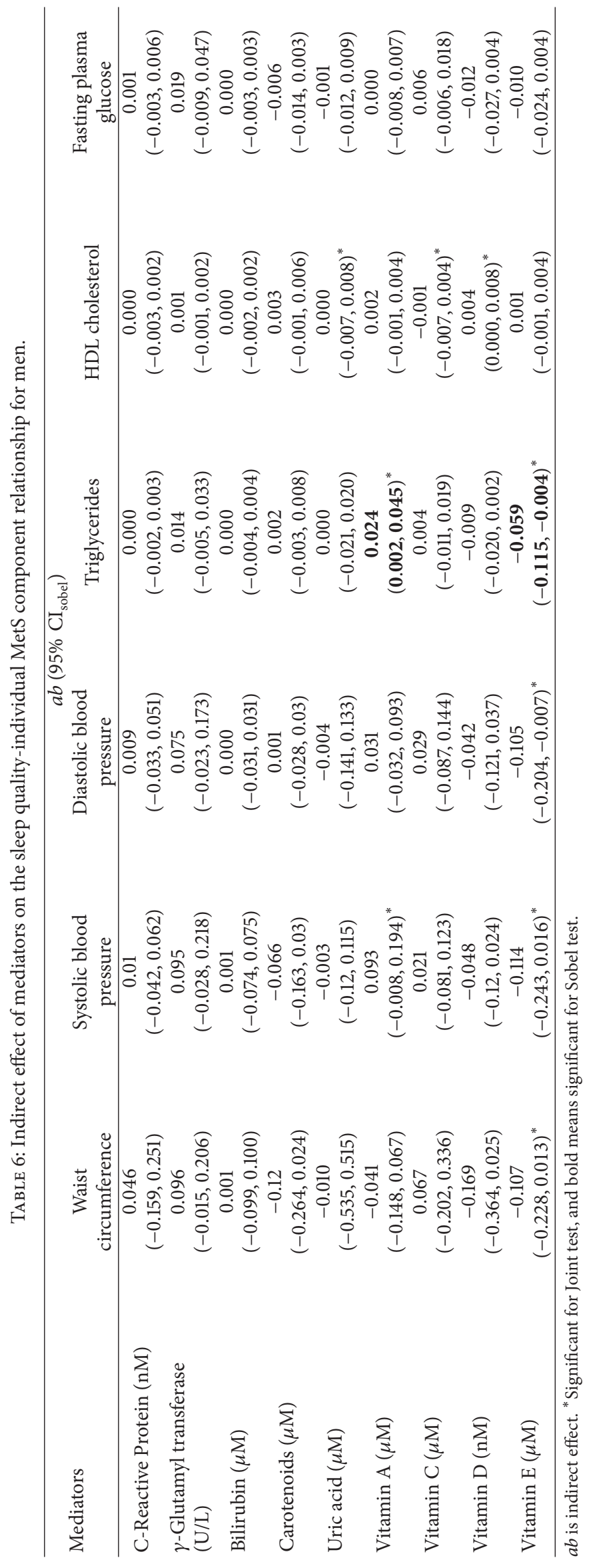


TABLE 7: Indirect effect of mediators on the sleep quality-individual MetS component relationship for women.

\begin{tabular}{|c|c|c|c|c|c|c|}
\hline \multirow[b]{2}{*}{ Mediators } & \multicolumn{6}{|c|}{$a b\left(95 \% \mathrm{CI}_{\text {sobel }}\right)$} \\
\hline & $\begin{array}{c}\text { Waist } \\
\text { circumference }\end{array}$ & $\begin{array}{l}\text { Systolic blood } \\
\text { pressure }\end{array}$ & $\begin{array}{l}\text { Diastolic blood } \\
\text { pressure }\end{array}$ & Triglycerides & HDL cholesterol & $\begin{array}{l}\text { Fasting plasma } \\
\text { glucose }\end{array}$ \\
\hline C-Reactive Protein (nM) & $\begin{array}{c}0.539 \\
(0.204,0.875)^{*}\end{array}$ & $\begin{array}{c}0.189 \\
(0.023,0.355)^{*}\end{array}$ & $\begin{array}{c}0.093 \\
(-0.005,0.190)\end{array}$ & $\begin{array}{c}0.017 \\
(0.000,0.034)^{*}\end{array}$ & $\begin{array}{c}-0.004 \\
(-0.009,0.001)\end{array}$ & $\begin{array}{c}0.030 \\
(0.011,0.048)\end{array}$ \\
\hline $\begin{array}{l}\gamma \text {-Glutamyl transferase } \\
(\mathrm{U} / \mathrm{L})\end{array}$ & $\begin{array}{c}0.143 \\
(-0.031,0.317)^{*}\end{array}$ & $\begin{array}{c}0.188 \\
(-0.006,0.382)\end{array}$ & $\begin{array}{c}0.031 \\
(-0.014,0.076)\end{array}$ & $\begin{array}{c}0.011 \\
(-0.004,0.026)\end{array}$ & $\begin{array}{c}0.000 \\
(-0.002,0.002)\end{array}$ & $\begin{array}{c}0.015 \\
(-0.004,0.034)\end{array}$ \\
\hline Bilirubin $(\mu \mathrm{M})$ & $\begin{array}{c}0.051 \\
(-0.059,0.161)\end{array}$ & $\begin{array}{c}0.002 \\
(-0.06,0.063)\end{array}$ & $\begin{array}{c}0.018 \\
(-0.025,0.060)\end{array}$ & $\begin{array}{c}0.004 \\
(-0.004,0.012)\end{array}$ & $\begin{array}{c}-0.002 \\
(-0.006,0.002)^{*}\end{array}$ & $\begin{array}{c}-0.002 \\
(-0.010,0.007)\end{array}$ \\
\hline Carotenoids $(\mu \mathrm{M})$ & $\begin{array}{c}0.199 \\
(0.001,0.397)\end{array}$ & $\begin{array}{c}0.133 \\
(-0.005,0.272)^{*}\end{array}$ & $\begin{array}{c}-0.019 \\
(-0.059,0.022)\end{array}$ & $\begin{array}{c}0.003 \\
(-0.001,0.008)\end{array}$ & $\begin{array}{c}-0.004 \\
(-0.009,0.000)\end{array}$ & $\begin{array}{c}0.012 \\
(-0.002,0.025)\end{array}$ \\
\hline Uric acid $(\mu \mathrm{M})$ & $\begin{array}{c}0.474 \\
(0.058,0.890)^{*}\end{array}$ & $\begin{array}{c}0.359 \\
(0.065,0.654)^{*}\end{array}$ & $\begin{array}{c}-0.022 \\
(-0.075,0.032)\end{array}$ & $\begin{array}{c}0.016 \\
(0.002,0.031)^{*}\end{array}$ & $\begin{array}{c}-0.003 \\
(-0.007,0.000)^{*}\end{array}$ & $\begin{array}{c}0.013 \\
(-0.002,0.028)^{*}\end{array}$ \\
\hline Vitamin $\mathrm{A}(\mu \mathrm{M})$ & $\begin{array}{c}0.073 \\
(-0.044,0.191)\end{array}$ & $\begin{array}{c}0.276 \\
(-0.049,0.602)\end{array}$ & $\begin{array}{c}-0.052 \\
(-0.145,0.042)\end{array}$ & $\begin{array}{c}0.022 \\
(-0.005,0.048)\end{array}$ & $\begin{array}{c}0.004 \\
(-0.001,0.009)\end{array}$ & $\begin{array}{c}0.000 \\
(-0.008,0.009)\end{array}$ \\
\hline Vitamin $\mathrm{C}(\mu \mathrm{M})$ & $\begin{array}{c}0.445 \\
(0.162,0.728)^{*}\end{array}$ & $\begin{array}{c}0.024 \\
(-0.199,0.246)\end{array}$ & $\begin{array}{c}0.127 \\
(0.020,0.234)^{*}\end{array}$ & $\begin{array}{c}0.014 \\
(0.003,0.025)^{*}\end{array}$ & $\begin{array}{l}-0.007 \\
(-0.013 \\
-0.002)\end{array}$ & $\begin{array}{c}0.017 \\
(0.001,0.034)^{*}\end{array}$ \\
\hline Vitamin D (nM) & $\begin{array}{c}0.140 \\
(-0.373,0.654)\end{array}$ & $\begin{array}{c}0.054 \\
(-0.234,0.342)\end{array}$ & $\begin{array}{c}0.006 \\
(-0.027,0.039)\end{array}$ & $\begin{array}{c}0.002 \\
(-0.006,0.011)\end{array}$ & $\begin{array}{c}-0.002 \\
(-0.007,0.004)^{*}\end{array}$ & $\begin{array}{c}0.004 \\
(-0.020,0.028)\end{array}$ \\
\hline Vitamin $\mathrm{E}(\mu \mathrm{M})$ & $\begin{array}{c}-0.015 \\
(-0.154,0.123)\end{array}$ & $\begin{array}{c}-0.006 \\
(-0.327,0.315)\end{array}$ & $\begin{array}{c}-0.001 \\
(-0.034,0.033)\end{array}$ & $\begin{array}{c}-0.005 \\
(-0.046,0.037)\end{array}$ & $\begin{array}{c}0.000 \\
(-0.001,0.001)\end{array}$ & $\begin{array}{c}-0.009 \\
(-0.040,0.022)\end{array}$ \\
\hline
\end{tabular}

$a b$ is indirect effect. ${ }^{*}$ Significant for Joint test, and bold means significant for Sobel test.

disturbances, even after adjusting for BMI [35]. Vitamin C supplementation, on the other hand, improved endothelial function in patients with obstructive sleep apnea [36], a condition associated with increased bodyweight [15]. In rodents, the beneficial effects of vitamin $\mathrm{C}$ on weight gain and the absorption of lipids has been demonstrated [37, 38], and some more limited research suggests that vitamin $C$ supplementation could help reduce adiposity through alterations in gene expression [39]. While the direct inflammatory and antioxidant mechanisms purported to influence the relationship between poor sleep quality and obesity remain unknown, our study provides initial evidence of a mediating role of CRP and vitamin C on the relationship between sleep quality and abdominal obesity.

4.2.2. Systolic Blood Pressure. While evidence suggests vitamin $C$ could improve endothelial function [36], we did not find that it significantly mediated the sleep quality-systolic blood pressure relationship; rather, we found vitamin A moderately mediated the sleep quality-systolic blood pressure relationship. In mice, deficiency in vitamin A decreased nonrapid eye movement (REM) sleep stages 3 and 4 (i.e., deep or slow wave sleep); and evidence in humans suggest that vitamin A has an important role in sleep homeostasis [40].

4.3. Sex-Stratified Indirect Mediation Effect. We found that CRP, GGT, carotenoids, uric acid, and vitamin C were moderate-to-large mediators of selected sleep-cardiometabolic health relationships in women only. Others have found similar sex differences between inflammation, oxidative stress, antioxidants, and cardiometabolic dysfunction [17, 41]. Increased systematic inflammation is a particular concern in aging women since poor sleep duration, poor sleep quality, lack of social interactions, and abdominal adiposity have all been associated with inflammation [42, 43]. Additionally, Okun et al. [44] demonstrated a link between inflammation and poor sleep quality, proposing that chronic low-grade inflammation as a result of poor sleep quality in early adulthood (i.e., 20s) may predispose women to inflammationrelated diseases in middle-adulthood. Indeed, the relationship between inflammation, oxidative stress, antioxidants, and age-related diseases warrants further study in women. Changes in female sex hormone levels, for instance, are associated with longer sleep duration and poorer sleep quality [45], while risk of cardiometabolic disease [46] and adiposity [47] also changes with circulating testosterone, estradiol, and sex hormone binding globulin levels. Taken together, our findings suggest that strategies to improve dietary or sleep habits may be valuable for the cardiometabolic health of women.

4.4. Strengths and Limitations. There are several study limitations that warrant mention. First, given the preliminary and cross-sectional nature of our findings, future longitudinal studies are needed to evaluate evidence for a cause-andeffect relationship. Second, in applying our study exclusion criteria, our final analytic sample was limited to less than $50 \%$ of the initial adult sample. Third, since all sleep quality variables were self-reported, they are susceptible to recall and response biases. However, we are not aware of a comprehensive population-based dataset that contains objective measures of sleep quality along with other physical measures necessary for this research question [i.e., accelerometry, polysomnography, or electroencephalogram, 
serum biomarkers, and cardiometabolic health information]. We were also unable to study the effect of adipokines, such as leptin and adiponectin, which have been linked to poor sleep quality [48] and obesity [49]. Moreover, this work is limited to a select number of biomarkers for inflammation and oxidative stress. Future work would therefore benefit from the inclusion of interleukin- 6 , tumour necrosis factor- $\alpha$, and malondialdehyde [50-52] in participants with diagnosed inflammatory diseases. Finally, since we used single measurements of exposure, outcome, and mediators, we are unable to account for potential changes in modifiable habits (i.e., sleep and diet) and their effect on our outcomes.

\section{Conclusions}

Improving sleep quality may minimize cardiometabolic decline though mechanisms involving inflammation, oxidative stress, and antioxidants. Further prospective work is needed to extend our understanding of the multiple pathways that may govern these factors.

\section{Conflict of Interests}

The authors declare that there is no conflict of interests regarding the publication of this paper.

\section{References}

[1] G. Atkinson and D. Davenne, "Relationships between sleep, physical activity and human health," Physiology and Behavior, vol. 90, no. 2-3, pp. 229-235, 2007.

[2] J. E. Ferrie, M. J. Shipley, F. P. Cappuccio et al., "A prospective study of change in sleep duration: associations with mortality in the Whitehall II cohort," Sleep, vol. 30, no. 12, pp. 1659-1666, 2007.

[3] J. Engeda, B. Mezuk, S. Ratliff, and Y. Ning, "Association between duration and quality of sleep and the risk of prediabetes: evidence from NHANES," Diabetic Medicine, vol. 30, no. 6, pp. 676-680, 2013.

[4] A. E. Mesas, P. Guallar-Castillón, E. López-García et al., "Sleep quality and the metabolic syndrome: the role of sleep duration and lifestyle," Diabetes/Metabolism Research and Reviews, vol. 30, no. 3, pp. 222-231, 2014

[5] H.-C. Hung, Y.-C. Yang, H.-Y. Ou, J.-S. Wu, F.-H. Lu, and C.J. Chang, "The association between self-reported sleep quality and metabolic syndrome," PLoS ONE, vol. 8, no. 1, Article ID e54304, 2013.

[6] E.-J. Lee, S.-G. Kang, J.-H. Shin, Y.-N. Hwang, K.-S. Ryu, and S.-W. Song, "Relationship between sleep quality and metabolic syndrome and inflammatory markers in middle-aged men in Korea," Korean Journal of Family Medicine, vol. 30, no. 5, pp. 344-351, 2009.

[7] J. B. Dowd, N. Goldman, and M. Weinstein, "Sleep duration, sleep quality, and biomarkers of inflammation in a Taiwanese population," Annals of Epidemiology, vol. 21, no. 11, pp. 799-806, 2011.

[8] W. Huang, S. Shah, Q. Long, A. K. Crankshaw, and V. Tangpricha, "Improvement of pain, sleep, and quality of life in chronic pain patients with vitamin D supplementation," Clinical Journal of Pain, vol. 29, no. 4, pp. 341-347, 2013.
[9] A. Emami Zeydi, Y. Jannati, H. Darvishi Khezri et al., "Sleep quality and its correlation with serum C-reactive protein level in hemodialysis patients," Saudi Journal of Kidney Diseases and Transplantation, vol. 25, no. 4, pp. 750-755, 2014.

[10] R. Liu, X. Liu, P. C. Zee et al., "Association between sleep quality and C-reactive protein: results from national health and nutrition examination survey, 2005-2008," PLOS ONE, vol. 9, no. 3, Article ID e92607, 2014.

[11] T. D. Singh, K. Patial, V. K. Vijayan, and K. Ravi, "Oxidative stress and obstructive sleep apnoea syndrome," The Indian Journal of Chest Diseases \& Allied Sciences, vol. 51, no. 4, pp. 217224, 2009.

[12] N. Khansari, Y. Shakiba, and M. Mahmoudi, "Chronic inflammation and oxidative stress as a major cause of age-related diseases and cancer," Recent Patents on Inflammation and Allergy Drug Discovery, vol. 3, no. 1, pp. 73-80, 2009.

[13] T. Kanagasabai and C. I. Ardern, "Contribution of inflammation, oxidative stress, and antioxidants to the relationship between sleep duration and cardiometabolic health," Sleep, In press.

[14] T. Yokoe, K. Minoguchi, H. Matsuo et al., "Elevated levels of Creactive protein and interleukin- 6 in patients with obstructive sleep apnea syndrome are decreased by nasal continuous positive airway pressure," Circulation, vol. 107, no. 8, pp. 11291134, 2003.

[15] A. Kanbay, E. Kaya, H. Buyukoglan et al., "Serum gammaglutamyl transferase activity is an independent predictor for cardiovascular disease in Obstructive Sleep Apnea Syndrome," Respiratory Medicine, vol. 105, no. 4, pp. 637-642, 2011.

[16] E. S. Ford, A. H. Mokdad, W. H. Giles, and D. W. Brown, "The metabolic syndrome and antioxidant concentrations: findings from the third national health and nutrition examination survey," Diabetes, vol. 52, no. 9, pp. 2346-2352, 2003.

[17] M. A. Beydoun, M. R. Shroff, X. Chen, H. A. Beydoun, Y. Wang, and A. B. Zonderman, "Serum antioxidant status is associated with metabolic syndrome among U.S. adults in recent national surveys," Journal of Nutrition, vol. 141, no. 5, pp. 903-913, 2011.

[18] D. A. Kenny, "Mediation," 2013, http://davidakenny.net/.

[19] AMA, "AMA Reference Ranges," 2014, http://www.amamanualofstyle.com/page/si-conversion-calculator.

[20] NCHS, "NHANES-About the National Health and Nutrition Examination Survey," 2013, http://www.cdc.gov/nchs/nhanes/ about_nhanes.htm.

[21] K. G. M. M. Alberti, R. H. Eckel, S. M. Grundy et al., "Harmonizing the metabolic syndrome: a joint interim statement of the International Diabetes Federation Task Force on Epidemiology and Prevention; National Heart, Lung, and Blood Institute; American Heart Association; World Heart Federation; International Atherosclerosis Society; and International Association for the Study of Obesity," Circulation, vol. 120, pp. 1640-1645, 2009.

[22] L. Plantinga, K. Lee, L. A. Inker et al., "Association of sleeprelated problems with CKD in the United States, 2005-2008," American Journal of Kidney Diseases, vol. 58, no. 4, pp. 554-564, 2011.

[23] NCHS, NHANES 2005-2006-Manuals, December 2013, http:// www.cdc.gov/nchs/nhanes/nhanes2005-2006/manuals05_06 .htm.

[24] T. Kanagasabai, J. X. Nie, C. Mason, and C. I. Ardern, "Metabolic syndrome and prevalent any-site, prostate, breast and colon cancers in the US adult population: NHANES 1999-2010," Journal of Metabolic Syndrome, vol. 3, article 135, 2014. 
[25] A.-C. Santos, S. Ebrahim, and H. Barros, "Alcohol intake, smoking, sleeping hours, physical activity and the metabolic syndrome," Preventive Medicine, vol. 44, no. 4, pp. 328-334, 2007.

[26] US Department of Health and Human Services, Physical Activity Guidelines for Americans, US Department of Health and Human Services, Washington, DC, USA, 2008.

[27] H.-H. Lin, P.-S. Tsai, S.-C. Fang, and J.-F. Liu, "Effect of kiwifruit consumption on sleep quality in adults with sleep problems," Asia Pacific Journal of Clinical Nutrition, vol. 20, no. 2, pp. 169174, 2011.

[28] G. Howatson, P. G. Bell, J. Tallent, B. Middleton, M. P. McHugh, and J. Ellis, "Effect of tart cherry juice (Prunus cerasus) on melatonin levels and enhanced sleep quality," European Journal of Nutrition, vol. 51, no. 8, pp. 909-916, 2012.

[29] L. Lavie, "Oxidative stress-a unifying paradigm in obstructive sleep apnea and comorbidities," Progress in Cardiovascular Diseases, vol. 51, no. 4, pp. 303-312, 2009.

[30] H. Hachul de Campos, L. Brandão, V. D’Almeida et al., "Sleep disturbances, oxidative stress and cardiovascular risk parameters in postmenopausal women complaining of insomnia," Climacteric, vol. 9, no. 4, pp. 312-319, 2006.

[31] G. Beccuti and S. Pannain, "Sleep and obesity," Current Opinion in Clinical Nutrition and Metabolic Care, vol. 14, no. 4, pp. 402412, 2011.

[32] I. Alam, K. Lewis, J. W. Stephens, and J. N. Baxter, "Obesity, metabolic syndrome and sleep apnoea: all pro-inflammatory states," Obesity Reviews, vol. 8, no. 2, pp. 119-127, 2007.

[33] L. M. Belalcazar, S. M. Haffner, W. Lang et al., "Lifestyle intervention and/or statins for the reduction of C-reactive protein in type 2 diabetes: from the look AHEAD study," Obesity, vol. 21, no. 5, pp. 944-950, 2013.

[34] G. Slater and J. Steier, "Excessive daytime sleepiness in sleep disorders," Journal of Thoracic Disease, vol. 4, no. 6, pp. 608-616, 2012.

[35] T. Liukkonen, P. Räsänen, A. Ruokonen et al., "C-reactive protein levels and sleep disturbances: observations based on the Northern Finland 1966 birth cohort study," Psychosomatic Medicine, vol. 69, no. 8, pp. 756-761, 2007.

[36] M. Grebe, H. J. Eisele, N. Weissmann et al., "Antioxidant vitamin C improves endothelial function in obstructive sleep apnea," American Journal of Respiratory and Critical Care Medicine, vol. 173, no. 8, pp. 897-901, 2006.

[37] K. M. Wasan, C. Zamfir, P. H. Pritchard, and R. A. Pederson, "Influence of phytostanol phosphoryl ascorbate (FM-VP4) on insulin resistance, hyperglycemia, plasma lipid levels, and gastrointestinal absorption of exogenous cholesterol in Zucker (fa/fa) fatty and lean rats," Journal of Pharmaceutical Sciences, vol. 92, no. 2, pp. 281-288, 2003.

[38] N. Ebine, I. Demonty, X. Jia, and P. J. H. Jones, "Plant stanol ascorbate esters reduce body weight gain through decreased energy absorption in hamsters," International Journal of Obesity, vol. 30, no. 5, pp. 751-757, 2006.

[39] J. Campión, F. I. Milargo, D. Fernández, and J. A. Martínez, "Diferential gene expression and adiposity reduction induced by ascorbic acid supplementation in a cafeteria model of obesity," Journal of Physiology and Biochemistry, vol. 62, no. 2, pp. 71-80, 2006.

[40] J. Ransom, P. J. Morgan, P. J. McCaffery, and P. N. Stoney, "The rhythm of retinoids in the brain," Journal of Neurochemistry, vol. 129, no. 3, pp. 366-376, 2014.
[41] C. S. Johnston, B. L. Beezhold, B. Mostow, and P. D. Swan, "Plasma vitamin C is inversely related to body mass index and waist circumference but not to plasma adiponectin in nonsmoking adults," Journal of Nutrition, vol. 137, no. 7, pp. 17571762, 2007.

[42] A. A. Prather, E. Puterman, E. S. Epel, and F. S. Dhabhar, "Poor sleep quality potentiates stress-induced cytokine reactivity in postmenopausal women with high visceral abdominal adiposity," Brain, Behavior, and Immunity, 2013.

[43] E. M. Friedman, M. S. Hayney, G. D. Love et al., "Social relationships, sleep quality, and interleukin-6 in aging women," Proceedings of the National Academy of Sciences of the United States of America, vol. 102, no. 51, pp. 18757-18762, 2005.

[44] M. L. Okun, M. Coussons-Read, and M. Hall, "Disturbed sleep is associated with increased C-reactive protein in young women," Brain, Behavior, and Immunity, vol. 23, no. 3, pp. 351354, 2009.

[45] M. F. Sowers, H. Zheng, H. M. Kravitz et al., "Sex steroid hormone profiles are related to sleep measures from polysomnography and the Pittsburgh sleep quality index," Sleep, vol. 31, no. 10, pp. 1339-1349, 2008.

[46] J. S. Brand, I. van der Tweel, D. E. Grobbee, M. H. EmmelotVonk, and Y. T. van der Schouw, "Testosterone, sex hormonebinding globulin and the metabolic syndrome: a systematic review and meta-analysis of observational studies," International Journal of Epidemiology, vol. 40, no. 1, Article ID dyq158, pp. 189-207, 2011.

[47] A. Tchernof and J.-P. Després, "Sex steroid hormones, sex hormone-binding globulin, and obesity in men and women," Hormone and Metabolic Research, vol. 32, no. 11-12, pp. 526-536, 2000.

[48] M. C. Mota, J. Waterhouse, D. A. De-Souza et al., "Sleep pattern is associated with adipokine levels and nutritional markers in resident physicians," Chronobiology International, vol. 31, no. 10, pp. 1130-1138, 2014.

[49] G. Fantuzzi, "Adipose tissue, adipokines, and inflammation," Journal of Allergy and Clinical Immunology, vol. 115, no. 5, pp. 911-920, 2005.

[50] E. Ho, K. Karimi Galougahi, C.-C. Liu, R. Bhindi, and G. A. Figtree, "Biological markers of oxidative stress: applications to cardiovascular research and practice," Redox Biology, vol. 1, no. 1, pp. 483-491, 2013.

[51] T. A. Pearson, G. A. Mensah, R. W. Alexander et al., "Markers of inflammation and cardiovascular disease. Application to clinical and public health practice: A statement for healthcare professionals from the centers for disease control and prevention and the American Heart Association," Circulation, vol. 107, no. 3, pp. 499-511, 2003.

[52] A. Williams and S. M. Scharf, "Obstructive sleep apnea, cardiovascular disease, and inflammation-is NF- $\kappa \mathrm{B}$ the key?" Sleep and Breathing, vol. 11, no. 2, pp. 69-76, 2007. 


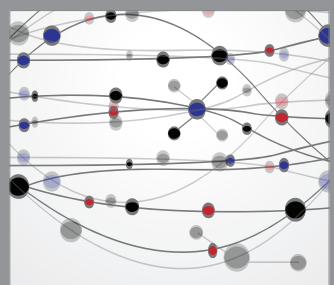

The Scientific World Journal
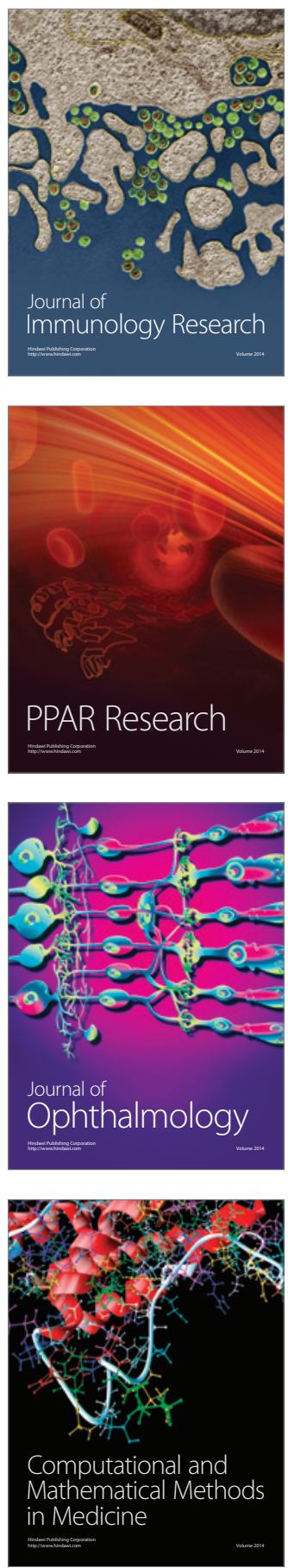

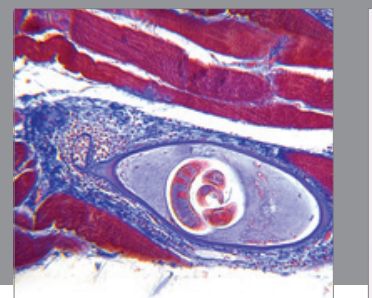

Gastroenterology

Research and Practice
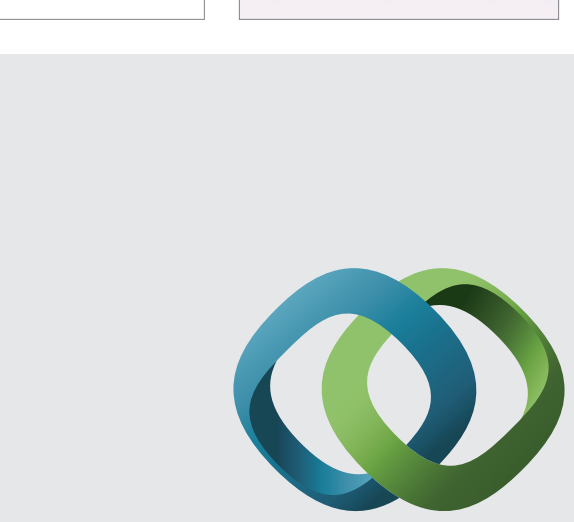

\section{Hindawi}

Submit your manuscripts at

http://www.hindawi.com
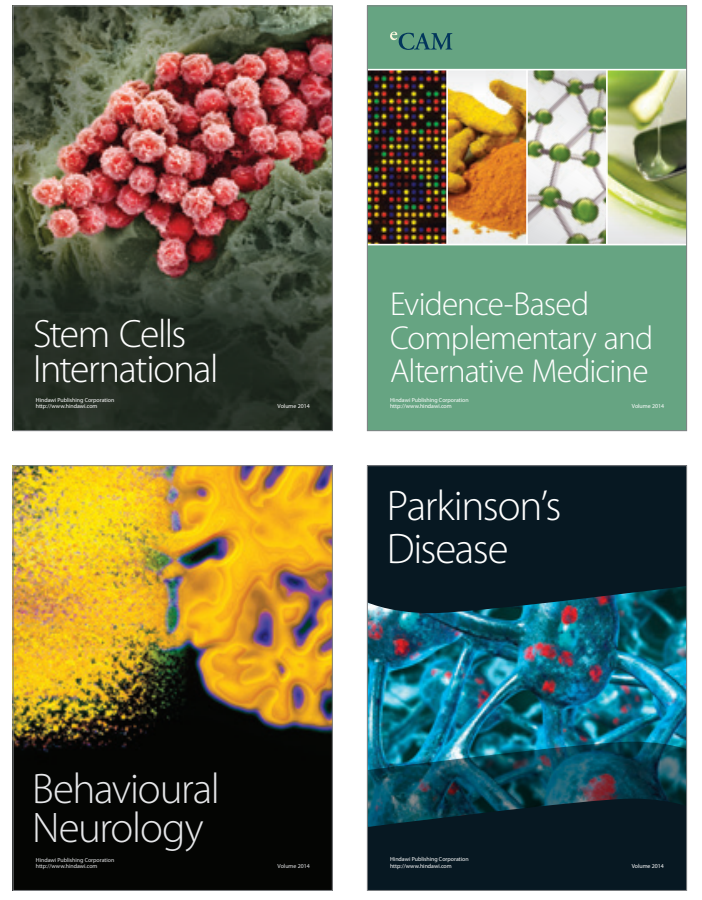
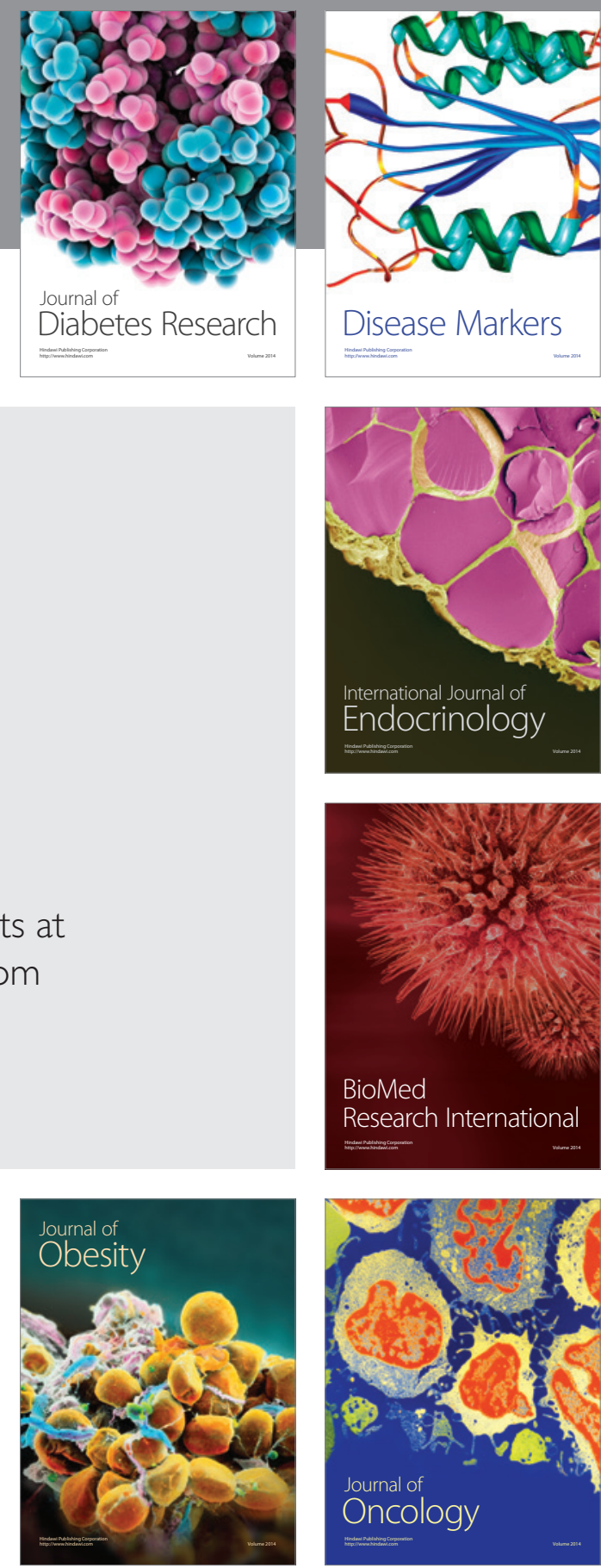

Disease Markers
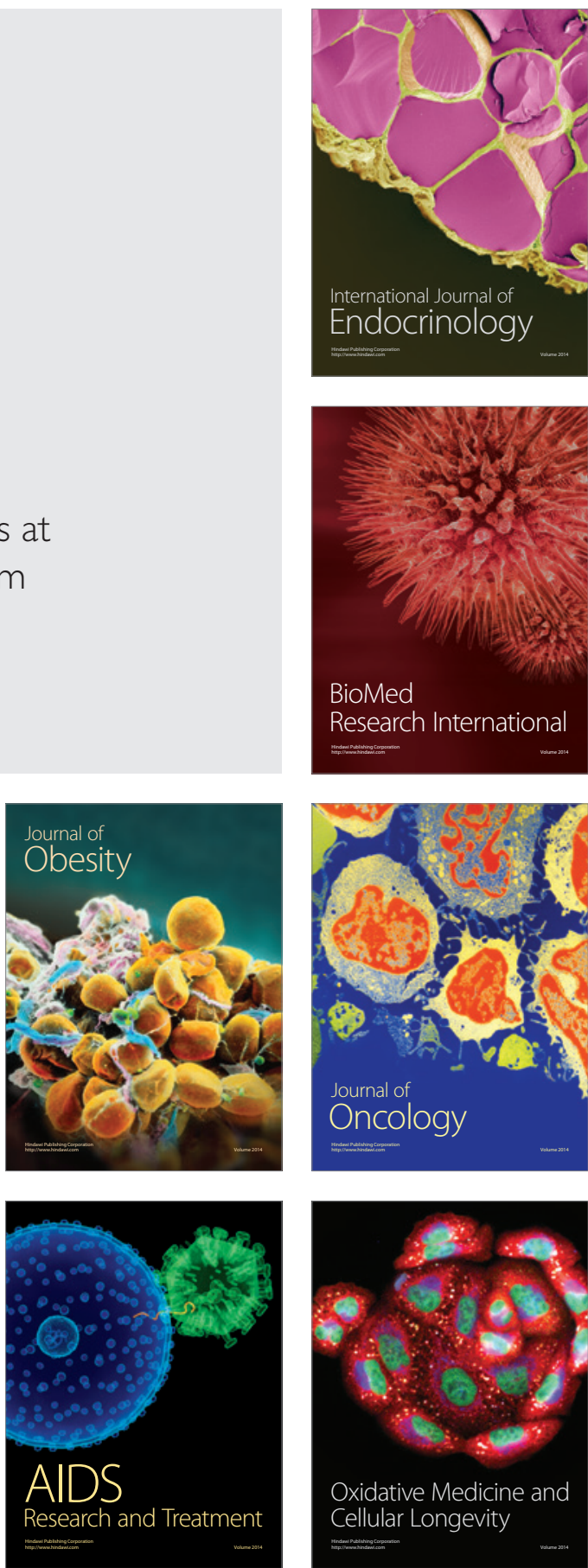